\title{
NON-TOEPLITZ DECAY BOUNDS FOR INVERSES OF HERMITIAN POSITIVE DEFINITE TRIDIAGONAL MATRICES*
}

\author{
ANDREAS FROMMER ${ }^{\dagger}$, CLAUDIA SCHIMMEL $^{\dagger}$, AND MARCEL SCHWEITZER ${ }^{\dagger}$
}

\begin{abstract}
It is well known that the entries of the inverse of a Hermitian positive definite, banded matrix exhibit a decay away from the main diagonal if the condition number of the matrix is not too large compared to the matrix size. There is a rich literature on bounds which predict and explain this decay behavior. However, all the widely known results on exponential decay lead to a Toeplitz matrix of bounds, i.e., they yield the same bound for all entries along a sub- or superdiagonal. In general, there is no reason to expect the inverse of $A$ to have a Toeplitz structure so that this is an obvious shortcoming of these decay bounds. We construct an example of a tridiagonal matrix for which the difference between these decay bounds and the actual decay is especially pronounced and then show how these bounds can be adapted to better reflect the actual decay by investigating certain (modified) submatrices of $A$. As a by-product, we also investigate how the distribution of all eigenvalues of $A$ rather than just the spectral interval influences the decay behavior. Here, our results hold for matrices with a general, not necessarily banded, sparsity structure.
\end{abstract}

Key words. matrix inverse, tridiagonal matrix, off-diagonal decay, Sherman-Morrison formula, Toeplitz matrix

AMS subject classifications. 15A16, 65F50, 65F60

1. Introduction and motivation. It has long been known that the entries of the inverse of a Hermitian positive definite, banded matrix decay away from the main diagonal. There is much literature devoted to this topic; see, e.g., $[10,11,13,19,23]$ and the references therein. Extensions to certain non-Hermitian matrices are discussed in [12, 14, 24, 25, 26]. Decay away from the diagonal is not specific to the inverse but also appears in other matrix functions. Due to its importance in many applications, there are in particular many publications dealing with the matrix exponential [4, 6, 18, 22] or general analytic functions [3, 27]. Recently, also other classes of functions like, e.g., Stieltjes functions, have been studied with respect to their decay behavior; see, e.g., [2, 5, 6, 15].

There is a wide variety of different areas in which these off-diagonal decay bounds are exploited. In [5, 9], knowledge of sharp decay bounds for the entries of matrix functions is used in order to design linearly scaling algorithms for matrix function computations. In several applications, e.g., in Markov chain queuing models [7, 8] and quantum dynamics [16], information on the decay behavior can be used for constructing sparse approximations for quantities of interest, thus reducing storage complexity and computational effort. In addition to this computationally-oriented usage of decay bounds, the knowledge of the decay behavior can also be exploited for theoretical purposes, e.g., in the stability analysis of finite element methods [1]. Of course, in all these applications, the efficiency of the designed algorithms and the quality of the obtained results strongly depend on how well the decay bounds predict the actual decay in the matrix function.

While oftentimes the decay behavior is captured very well by the classical bounds-and they can be shown to be sharp in certain cases (see [11, Section 3]) — there are also situations where the bounds are quite misleading. To illustrate this, we first recall the classical bound for

*Received December 19, 2017. Accepted June 25, 2018. Published online on October 11, 2018. Recommended by R. Nabben. This work is supported by Deutsche Forschungsgemeinschaft (DFG) within SFB/TRR55 "Hadron Physics from Lattice QCD".

${ }^{\dagger}$ School of Mathematics and Natural Sciences, Bergische Universität Wuppertal, 42097 Wuppertal, Germany (\{frommer, schimmel, schweitzer\}@math.uni-wuppertal.de). 

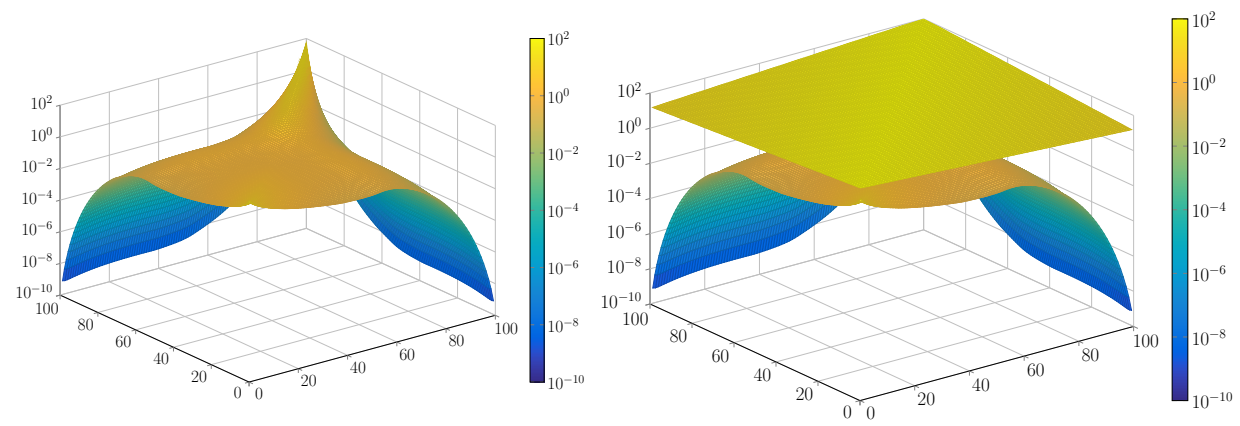

FIG. 1.1. Magnitude of the entries of $A^{-1}$ (left) and $Q$ from (1.3) (right), where $A$ is the matrix from Example 1.2.

the matrix inverse from [11]. We say that $A$ is $\beta$-banded if $A_{i, j}=0$ for $|i-j|>\beta$ (i.e., a tridiagonal matrix is 1-banded, a pentadiagonal matrix is 2-banded etc.).

THEOREM 1.1 (Theorem 2.4 in [11]). Let $A$ be Hermitian positive definite and $\beta$ banded with smallest eigenvalue $\lambda_{\min }$, largest eigenvalue $\lambda_{\max }$, and condition number $\kappa(A)=\lambda_{\max } / \lambda_{\min }$. Then,

$$
\left|\left[A^{-1}\right]_{i, j}\right| \leq c q^{\frac{|i-j|}{\beta}}
$$

with

$$
q=\frac{\sqrt{\kappa(A)}-1}{\sqrt{\kappa(A)}+1} \quad \text { and } \quad c=\max \left\{\frac{1}{\lambda_{\min }}, \frac{(1+\sqrt{\kappa(A)})^{2}}{2 \lambda_{\max }}\right\} .
$$

Typically, the quality of the decay bounds is illustrated by showing the actual decay in one row or column of the inverse and comparing it to the bound for this row or column. In the example below, we instead compare the whole matrix $A^{-1}$ to the matrix containing all the bounds (1.1). If we apply Theorem 1.1 to a tridiagonal matrix, i.e., $\beta=1$, then collecting all the bounds (1.1) in a matrix $Q$ yields the symmetric Toeplitz structure

$$
Q=c \cdot\left[\begin{array}{ccccc}
q^{0} & q^{1} & \cdots & \cdots & q^{n-1} \\
q^{1} & q^{0} & q^{1} & \ddots & \vdots \\
\vdots & q_{1} & \ddots & \ddots & \vdots \\
\vdots & \ddots & \ddots & \ddots & q^{1} \\
q^{n-1} & \cdots & \cdots & q^{1} & q^{0}
\end{array}\right]
$$

which, by Theorem 1.1, fulfills

$$
Q \geq\left|A^{-1}\right|,
$$

where " $\geq$ " and "| $\cdot \mid$ " are understood componentwise. Similarly, all the results on decay bounds for banded matrices from $[2,5,6,14,15,24,25,26]$ also result in a Toeplitz-structured matrix $Q$ of bounds. See also [12] for Toeplitz type bounds for block-tridiagonal matrices. However, even when $A$ is a Toeplitz matrix, $A^{-1}$ is in general not Toeplitz, indicating that the bound (1.4) will typically not estimate all the entries of $A^{-1}$ equally well when going along a specific sub- or superdiagonal. We now give an example where this effect is particularly pronounced. 
EXAMPLE 1.2. We construct a symmetric, tridiagonal matrix with prescribed spectrum following the construction principle developed in [20, Section 6.1]. The idea is to start with a diagonal matrix containing the prescribed eigenvalues and then apply a series of (twosided) Givens rotations in order to introduce nonzero elements on the sub- and superdiagonal. Nonzero elements that are introduced outside the band are immediately chased off the bottomright corner of the matrix, similarly to Schwarz band reduction [29].

Specifically, we construct a symmetric positive definite tridiagonal matrix $A \in \mathbb{R}^{100 \times 100}$ with logarithmically spaced eigenvalues in the interval $\left[10^{-2}, 10^{2}\right]$. Figure 1.1 displays the magnitude of the entries of $A^{-1}$ and of the Toeplitz matrix (1.3) containing the bounds from Theorem 1.1. Two problems with the bound are apparent: On the one hand, the magnitude of the entries is very severely overestimated, and on the other hand, the structure of $A^{-1}$ is far from being Toeplitz in contrast to the matrix $Q$.

In the remainder of this paper we develop a theoretical framework for explaining and accurately predicting a decay behavior as observed in Example 1.2. In Section 2, we very briefly explain how the computation of decay bounds is related to polynomial approximation problems as this relation is needed later on for constructing our refined bounds. In Section 3, we show how to obtain a family of decay bounds based on full spectral information of $A$, while the bound in Theorem 1.1 is only based on the largest and smallest eigenvalue, i.e., the spectral interval. Although this information is typically not available in practical situations, it will help us make a first step towards explaining the decay behavior observed for the matrix $A$ from Example 1.2. As these bounds turn out to be still not necessarily accurate, we improve them further in Section 4 by relating the decay above and below the diagonal of the $k$ th column of $A^{-1}$ to the eigenvalues of (slightly modified) $k \times k$ and $(n-k) \times(n-k)$ submatrices of $A$, respectively. By combining the approaches from Sections 3 and 4, we obtain sharp bounds for the entries of $A^{-1}$ even in extreme situations as the one from Example 1.2. Concluding remarks are given in Section 5.

2. The relation between off-diagonal decay in banded matrices and polynomial approximation. In this section, we briefly outline the connection between the errors of polynomial approximations and decay bounds for entries of matrix inverses, which is, e.g., also the basis of the bounds cited in Theorem 1.1. We focus only on the Hermitian positive definite, banded case, as only this is relevant for the later developments in this paper.

For a Hermitian positive definite, $\beta$-banded matrix $A \in \mathbb{C}^{n \times n}$, one exploits the knowledge of the error

$$
\varepsilon_{\mathbb{E}}(m):=\max _{z \in \mathbb{E}}\left|z^{-1}-p_{m}(z)\right|,
$$

where $\mathbb{E} \subset \mathbb{C}$ is a set containing $\sigma(A)$, the spectrum of $A$, and $p_{m}$ is a polynomial approximation of $z^{-1}$ of degree at most $m$. Using the eigendecomposition $A=U \Lambda U^{H}$ with $\Lambda$ diagonal and $U$ unitary, we see that the relation

$$
\begin{aligned}
\left\|A^{-1}-p_{m}(A)\right\|_{2} & =\left\|U\left(\Lambda^{-1}-p_{m}(\Lambda)\right) U^{H}\right\|_{2}=\left\|\Lambda^{-1}-p_{m}(\Lambda)\right\|_{2} \\
& =\max _{z \in \sigma(A)}\left|z^{-1}-p_{m}(z)\right|
\end{aligned}
$$

holds, and it actually holds for any normal matrix $A$. Now, for bounding an entry $\left|\left[A^{-1}\right]_{i, j}\right|$, write $|i-j|=m \beta+s$ with $m \geq 0$ and $s \in\{1, \ldots, \beta\}$, i.e., $m$ is the largest number for which $|i-j|>m \beta$. Since for every polynomial of degree $m$ the matrix $p_{m}(A)$ has bandwidth $m \beta$, we have $\left[p_{m}(A)\right]_{i, j}=0$ for this choice of $m$. Putting this together yields

$$
\begin{aligned}
\left|\left[A^{-1}\right]_{i, j}\right| & =\left|\left[A^{-1}\right]_{i, j}-\left[p_{m}(A)\right]_{i, j}\right| \leq\left\|A^{-1}-p_{m}(A)\right\|_{2} \\
& =\max _{z \in \sigma(A)}\left|z^{-1}-p_{m}(z)\right| \leq \max _{z \in \mathbb{E}}\left|z^{-1}-p_{m}(z)\right|=\varepsilon_{\mathbb{E}}(m),
\end{aligned}
$$


i.e., the polynomial approximation error $\varepsilon_{\mathbb{E}}(m)$ gives an upper bound for all values $\left|\left[A^{-1}\right]_{i, j}\right|$ with $|i-j|>m \beta$. Note that in the Hermitian positive definite case, one can take $\mathbb{E}=\left[\lambda_{\min }(A), \lambda_{\max }(A)\right]$. Within this approach, the best possible decay bounds are then obtained by taking the polynomial $p_{m}$ which solves the min-max problem

$$
\min _{p \in \Pi_{m}} \max _{z \in\left[\lambda_{\min }(A), \lambda_{\max }(A)\right]}\left|z^{-1}-p_{m}(z)\right| .
$$

3. Superlinear decay bounds based on full spectral information on $\boldsymbol{A}$. The decay bounds from Theorem 1.1 are obtained by taking $\mathbb{E}=\left[\lambda_{\min }(A), \lambda_{\max }(A)\right]$ in the approach outlined in Section 2 and then using the best polynomial approximation for the inverse on a real positive interval. A drawback of the bounds of Theorem 1.1 is that they cannot accurately capture the actual decay behavior if the decay is superlinear. This problem is comparable to what one observes for the classical textbook convergence bound for the conjugate gradient method; see, e.g., [28]. While for a given condition number one can always find a matrix such that the bound for step $k$ is sharp, it is typically neither sharp for other matrices with the same condition number, nor for other steps of the iteration. In the same way, one cannot expect the decay bounds from Theorem 1.1 to be sharp for all matrices with a given condition number or even just for all entries of one specific matrix. In particular, the classical CG convergence result only predicts linear convergence (and similarly, the bound of Theorem 1.1 only predicts linear decay), while in practice one often observes superlinear convergence due to spectral adaptation.

A simple approach for explaining the superlinear convergence is based on bounding the iteration polynomial from the CG method by other, so-called composite polynomials, which leads to a family of bounds. This approach is described in detail in [21, Chapter 5.6.4] and is adumbrated in the proof of Theorem 3.1 below.

To use the same idea, we cannot use the bounds of Theorem 1.1 but have to resort to a slightly different approach: Instead of using a polynomial approximation for the inverse directly, we use shifted and normalized Chebyshev polynomials similarly to what was done in [15] for general line segments in the complex plane. In the case of a Hermitian positive definite matrix, this gives the same decay rate as that of Theorem 1.1 with a new constant $C$ which fulfills $C \leq 2 c$, i.e., the new constant can be slightly worse than the constant $c$ in (1.2). The advantage is that with this approach we may use the same idea as for the CG convergence analysis to explain a superlinear convergence behavior. Altogether this gives the following family of bounds based on the effective condition number.

THEOREM 3.1. Let $A \in \mathbb{C}^{n \times n}$ be Hermitian positive definite and $\beta$-banded with eigenvalues $\lambda_{\min }(A)=\lambda_{1} \leq \lambda_{2} \leq \cdots \leq \lambda_{n}=\lambda_{\max }(A)$. Further, let

$$
\kappa_{\ell}(A)=\frac{\lambda_{n-\ell}}{\lambda_{1}}, \quad q_{\ell}=\frac{\sqrt{\kappa_{\ell}(A)}-1}{\sqrt{\kappa_{\ell}(A)}+1}, \quad \text { and } \quad C=\frac{2}{\lambda_{1}} .
$$

Then the entries of $A^{-1}$ can be bounded as

$$
\left|\left[A^{-1}\right]_{i, j}\right| \leq C q_{\ell}^{\frac{|i-j|}{\beta}-\ell} \quad \text { for all } \ell=0,1, \ldots,\left\lfloor\frac{|i-j|}{\beta}\right\rfloor .
$$

Proof. Instead of bounding the entries of the inverse by using the polynomial best approximation on an interval containing the spectrum of $A$, we now first work with the discrete 
set $\sigma(A)=\left\{\lambda_{1}, \ldots, \lambda_{n}\right\}$. Because of (2.1), we can estimate the entries of the inverse as

$$
\begin{aligned}
& \left|\left[A^{-1}\right]_{i, j}\right| \leq \min _{p_{m} \in \Pi_{m}} \max _{z \in\left\{\lambda_{1}, \ldots, \lambda_{n}\right\}}\left|z^{-1}-p_{m}(z)\right|=\min _{\substack{p_{m+1} \in \Pi_{m+1} \\
p_{m+1}(0)=1}} \max _{z \in\left\{\lambda_{1}, \ldots, \lambda_{n}\right\}}\left|\frac{p_{m+1}(z)}{z}\right| \\
& \leq \frac{1}{\lambda_{1}} \min _{\substack{p_{m+1} \in \Pi_{m+1} \\
p_{m+1}(0)=1}} \max _{z \in\left\{\lambda_{1}, \ldots, \lambda_{n}\right\}}\left|p_{m+1}(z)\right| \\
& \leq \frac{1}{\lambda_{1}} \min _{\begin{array}{c}
p_{m+1-\ell} \in \Pi_{m+1-\ell} \\
p_{m+1-\ell}(0)=1
\end{array}} \max _{z \in\left\{\lambda_{1}, \ldots, \lambda_{n}\right\}}\left|r_{\ell}(z) p_{m+1-\ell}(z)\right|,
\end{aligned}
$$

where

$$
r_{\ell}(z)=\prod_{i=n-l+1}^{n}\left(1-\frac{z}{\lambda_{i}}\right)
$$

is a polynomial which satisfies $r_{\ell}(0)=1, r_{\ell}\left(\lambda_{i}\right)<1$ for $i=1, \ldots, n-\ell$, and $r_{\ell}\left(\lambda_{i}\right)=0$ for $i=n-\ell+1, \ldots, n$. Therefore, the entries of the inverse can further be bounded via

$$
\begin{aligned}
& \frac{1}{\lambda_{1}} \min _{\substack{p_{m+1-\ell} \in \Pi_{m+1-\ell} \\
p_{m+1-\ell}(0)=1}} \max _{z \in\left\{\lambda_{1}, \ldots, \lambda_{n-\ell}\right\}}\left|r_{\ell}(z) p_{m+1-\ell}(z)\right| \\
& \leq \frac{1}{\lambda_{1}} \max _{z \in\left\{\lambda_{1}, \ldots, \lambda_{n-\ell}\right\}}\left|r_{\ell}(z)\right| \min _{\substack{p_{m+1-\ell} \in \Pi_{m+1-\ell} \\
p_{m+1-\ell}(0)=1}} \max _{z \in\left\{\lambda_{1}, \ldots, \lambda_{n-\ell}\right\}}\left|p_{m+1-\ell}(z)\right| \\
& \leq \frac{1}{\lambda_{1}} \min _{p_{m+1-\ell} \in \Pi_{m+1-\ell}} \max _{z \in\left[\lambda_{1}, \lambda_{n-\ell}\right]}\left|p_{m+1-\ell}(z)\right| \\
& =\frac{1}{\lambda_{1}} \max _{z \in\left[\lambda_{1}, \lambda_{n-\ell}\right]}\left|T_{m+1-\ell}(z)\right|,
\end{aligned}
$$

where $T_{m+1-\ell}(z)$ is the normalized Chebyshev polynomial of degree $m+1-\ell$ for the interval $\left[\lambda_{1}, \lambda_{n-\ell}\right]$. Now, it is well-known [28] that

$$
\max _{z \in\left[\lambda_{1}, \lambda_{n-\ell}\right]}\left|T_{m+1-\ell}(z)\right| \leq 2 q_{\ell}^{m+1-\ell}
$$

so that (3.2) follows by using $|i-j| / \beta \leq m+1$.

The family (3.2) of bounds can potentially predict the decay behavior in $A^{-1}$ much more accurately than (1.1) - which is contained as a special case for $\ell=0$ (except for a factor 2 in the constant) - if one chooses the value of $\ell$ which minimizes (3.2) for each entry $\left[A^{-1}\right]_{i, j}$.

EXAMPLE 3.2. We consider the matrix from Example 1.2 again, but this time we define a matrix $Q$ such that

$$
Q_{i, j}=\min _{\ell=0, \ldots,\left\lfloor\frac{\lfloor i-j \mid}{\beta}\right\rfloor} C q_{\ell}^{\frac{|i-j|}{\beta}-\ell},
$$

where $q_{\ell}$ and $C$ are defined in (3.1). Note that the choice of $\ell$ that minimizes the right-hand side of (3.3) depends on the distribution of the eigenvalues of $A$. A larger number $\ell$ improves the effective condition number and therefore the decay rate $q_{\ell}$, but at the same time the exponent $\frac{|i-j|}{\beta}-\ell$ decreases. Therefore, the distribution of the eigenvalues determines whether the improvement of the decay rate counterbalances the smaller exponent. 

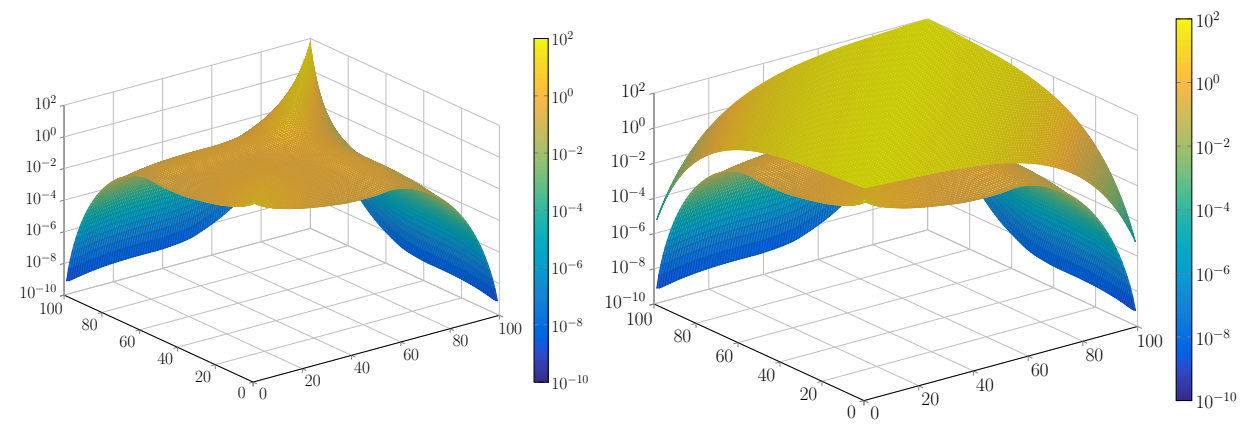

FIG. 3.1. Magnitude of the entries of $A^{-1}$ (left) and $Q$ from (3.3) (right) on a logarithmic scale, where $A$ is the matrix from Example 1.2.
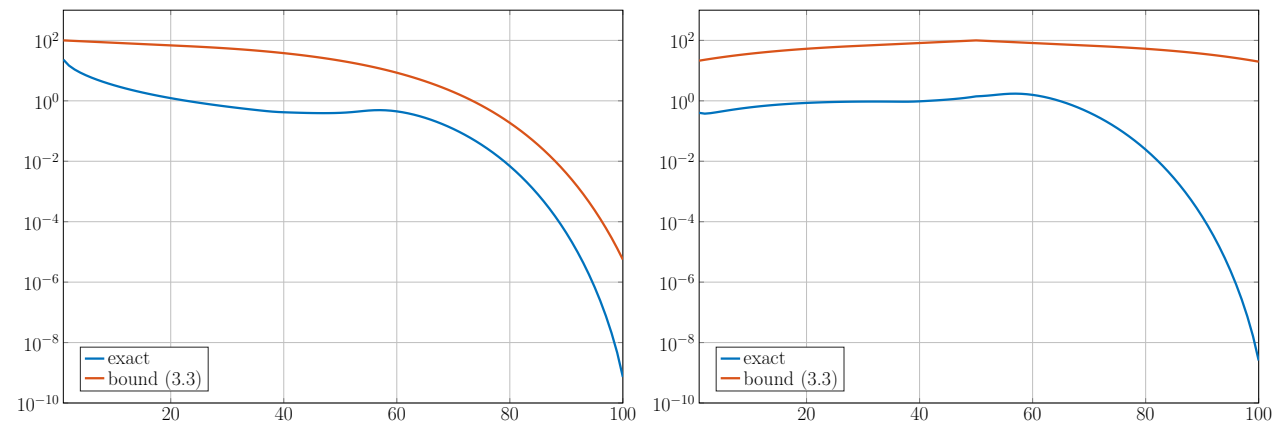

FIG. 3.2. Magnitude of the entries of the first (left) and 50th (right) column of $A^{-1}$ and the corresponding bounds (3.3) on a logarithmic scale, where A is the matrix from Example 1.2.

The magnitude of the entries of the resulting matrix $Q$ is given in Figure 3.1 (together with the magnitude of the entries of $A^{-1}$ for comparison). In contrast to what we observed in Figure 1.1, the entries are not overestimated as much as before (though still by several orders of magnitude), and at least in the first few rows and columns (which correspond to the front part of the plotted surfaces), the qualitative decay behavior is predicted quite accurately. For the other rows and columns, however, the decay behavior predicted by the bounds is still not satisfactory. To better understand why this is the case, we take a closer look at the decay behavior in two individual columns in Figure 3.2. On the left-hand side, the magnitude of the entries of the first column of $A^{-1}$ and the corresponding bounds are given, and on the right-hand side, the same information is shown for the 50th column. The plot on the right-hand side nicely illustrates the main problem that still occurs with (3.3): While the bounds obtained by (3.3) give a better idea of the actual decay behavior, they still lead to a symmetric Toeplitz structure of $Q$. As a consequence, the bound for the 50th column in the right part of Figure 3.2 is symmetric with respect to the 50th entry, and the bounds for the first fifty entries of the first column plotted in the left part of Figure 3.2 agree with the bounds for the entries 51-100 of the 50th column. This means in particular that the decay predicted "above" the diagonal (i.e., for $i<j$ ) is the same as the decay predicted "below" the diagonal (i.e., for $i>j$ ), although the actual decay is very different for these two parts of the 50th column. Whenever this is the case, any symmetric Toeplitz-structured bound has this shortcoming as it must be valid for both parts of the column, and will thus be forced to follow the part of the column with the "slower decay".

It is important to notice that the approach presented in this section can immediately be generalized to Hermitian and positive definite matrices with an arbitrary, not necessarily 
banded, sparsity pattern. All one has to do is to replace the quantity $|i-j| / \beta$ by the distance $\mathrm{d}(i, j)$ of the vertices $i$ and $j$ in the undirected graph $G(A)=(V, E)$ induced by $A$ with vertices $V=\{1, \ldots, n\}$ and edges $E=\left\{\{i, j\}: i \neq j\right.$ and $\left.a_{i j} \neq 0\right\}$. Everything then works in a completely analogous manner by using the fact that in a polynomial $p_{m}(A)$ of degree $m$ the $(i, j)$-entry is zero if $\mathrm{d}(i, j)>m$ (see, e.g., $[6,15])$ so that we can state the following result without proof.

THEOREM 3.3. Let the matrix $A \in \mathbb{C}^{n \times n}$ be Hermitian positive definite, and let denote $d(i, j)$ the distance of the vertices $i$ and $j$ in its graph $G(A)$. Let $\lambda_{\min }(A)=\lambda_{1} \leq \lambda_{2} \leq$ $\cdots \leq \lambda_{n}=\lambda_{\max }(A)$ be the eigenvalues of $A$, and let $\kappa_{\ell}(A), q_{\ell}$, and $C=\frac{2}{\lambda_{1}}$ be defined as in Theorem 3.1. Then the entries of $A^{-1}$ can be bounded as

$$
\left|\left[A^{-1}\right]_{i, j}\right| \leq C q_{\ell}^{d(i, j)-\ell} \quad \text { for all } \ell=0,1, \ldots, d(i, j) .
$$

Motivated by Example 3.2, we now proceed to show how to obtain bounds that are not restricted to a Toeplitz structure. In doing so, we use the bound of Theorem 3.1 for submatrices of $A$.

4. Decay bounds based on a block-partitioning of $\boldsymbol{A}$. This section contains our second main result, which relates the decay in $A^{-1}$ to the decay of the matrices in a block-partitioning of $A$ when $A$ is tridiagonal. The basic idea is to perform a rank-one modification of $A$ that reduces it to a block-diagonal form (similar to what is done in the divide and conquer algorithm for the symmetric tridiagonal eigenvalue problem [17]). By applying the Sherman-Morrison formula [30], the inverse of $A$ can then be written as the sum of the inverse of a block-diagonal matrix and a rank-one matrix. By exploiting the fact that both the inverse of the block-diagonal matrix and the rank-one term exhibit off-diagonal decay, the result then follows. So fix $k \in\{1, \ldots, n-1\}$ and decompose

$$
A=\left[\begin{array}{ll}
A_{11} & A_{22} \\
A_{21} & A_{22}
\end{array}\right]=\left[\begin{array}{cc}
B_{1} & 0 \\
0 & B_{2}
\end{array}\right]+\boldsymbol{u u}^{H}, \quad \boldsymbol{u}=\alpha\left(\boldsymbol{e}_{k}+\frac{a_{k+1, k}}{\left|a_{k+1, k}\right|} \boldsymbol{e}_{k+1}\right) \in \mathbb{C}^{n}
$$

with $\alpha=\sqrt{\left|a_{k+1, k}\right|}$ and $\boldsymbol{e}_{k}, \boldsymbol{e}_{k+1}$ the $k$ th and $(k+1)$ st canonical unit vectors in $\mathbb{C}^{n}$, respectively. Note that $B_{1} \in \mathbb{C}^{k \times k}$ is tridiagonal and that it differs from $A_{11}$ only in its $(k, k)$ entry, which is $\left[B_{1}\right]_{k, k}=a_{k, k}-\left|a_{k+1, k}\right|$. Similarly, $B_{2} \in \mathbb{C}^{(n-k) \times(n-k)}$ is tridiagonal, too, and it differs from $A_{22}$ only in its $(1,1)$ entry, which is $\left[B_{2}\right]_{1,1}=a_{k+1, k+1}-\left|a_{k+1, k}\right|$.

THEOREM 4.1. Let $A \in \mathbb{C}^{n \times n}$ be a tridiagonal Hermitian positive definite matrix. Assume that $B_{1}$ and $B_{2}$ in (4.1) are positive definite, and define for $s=1,2$

$$
\kappa_{s}=\frac{\lambda_{\max }\left(B_{s}\right)}{\lambda_{\min }\left(B_{s}\right)}, \quad q_{s}=\frac{\sqrt{\kappa_{s}}-1}{\sqrt{\kappa_{s}+1}}, \quad c_{s}=\frac{2}{\lambda_{\min }\left(B_{s}\right)} .
$$

Then the entries of $A^{-1}$ can be bounded as

$$
\left|\left[A^{-1}\right]_{i, j}\right| \leq \begin{cases}c_{1} q_{1}^{|i-j|}+c_{1}^{2} \widetilde{c} q_{1}^{2 k-j-i} & \text { for } i, j \leq k, \\ c_{2} q_{2}^{|i-j|}+c_{2}^{2} \widetilde{c} q_{2}^{i+j-2(k+1)} & \text { for } i, j>k, \\ c_{1} c_{2} \widetilde{c} q_{1}^{k-i} q_{2}^{j-k-1} & \text { for } i \leq k<j, \\ c_{1} c_{2} \widetilde{c} q_{1}^{j-k} q_{2}^{i-k-1} & \text { for } j \leq k<i,\end{cases}
$$

with the constant

$$
\widetilde{c}=\frac{\left|a_{k+1, k}\right|}{1+\left|a_{k+1, k}\right|\left(\frac{1}{\lambda_{\max }\left(B_{1}\right)}+\frac{1}{\lambda_{\max }\left(B_{2}\right)}\right)} .
$$


Proof. Let $B=\operatorname{diag}\left(B_{1}, B_{2}\right)$. The Sherman-Morrison formula gives

$$
A^{-1}=B^{-1}-\frac{B^{-1} \boldsymbol{u} \boldsymbol{u}^{H} B^{-1}}{1+\boldsymbol{u}^{H} B^{-1} \boldsymbol{u}}=: B^{-1}-R .
$$

Obviously, we thus have

$$
\left|\left[A^{-1}\right]_{i, j}\right| \leq\left|\left[B^{-1}\right]_{i, j}\right|+\left|R_{i, j}\right|,
$$

and as $B^{-1}=\operatorname{diag}\left(B_{1}^{-1}, B_{2}^{-1}\right)$, we can use Theorem 3.1 for $\ell=0$ applied to $B_{1}$ and $B_{2}$ in order to bound the entries of $B^{-1}$ as

$$
\left|\left[B^{-1}\right]_{i, j}\right|= \begin{cases}\left|\left[B_{1}^{-1}\right]_{i, j}\right| \leq c_{1} q_{1}^{|i-j|} & \text { for } i, j \leq k \\ \left|\left[B_{2}^{-1}\right]_{i, j}\right| \leq c_{2} q_{2}^{|i-j|} & \text { for } i, j>k \\ 0 & \text { otherwise }\end{cases}
$$

Let $M_{\bullet} \ell$ denote the $\ell$-th column of a matrix $M$, we have

$$
B^{-1} \boldsymbol{u}=\alpha\left[\begin{array}{c}
{\left[B_{1}^{-1}\right]_{\bullet}, k} \\
\frac{a_{k+1, k}}{\left|a_{k+1, k}\right|}\left[B_{2}^{-1}\right] \bullet, 1
\end{array}\right],
$$

which shows that the absolute values of the entries of the rank-one term $R$ are given by

$$
\left|R_{i, j}\right|=\frac{\alpha^{2}}{1+\boldsymbol{u}^{H} B^{-1} \boldsymbol{u}} \cdot\left\{\begin{array}{l}
\left|\left[B_{1}^{-1}\right]_{i, k}\right| \cdot\left|\left[B_{1}^{-1}\right]_{j, k}\right| \leq c_{1}^{2} q_{1}^{k-i} q_{1}^{k-j} \quad \text { for } i, j \leq k, \\
\left|\left[B_{2}^{-1}\right]_{i-k, 1}\right| \cdot\left|\left[B_{2}^{-1}\right]_{j-k, 1}\right| \leq c_{2}^{2} q_{2}^{i-k-1} q_{1}^{j-k-1} \text { for } i, j>k, \\
\left|\left[B_{1}^{-1}\right]_{i, k}\right| \cdot\left|\left[B_{2}^{-1}\right]_{j-k, 1}\right| \leq c_{1} c_{2} q_{1}^{k-i} q_{2}^{j-k-1} \text { for } i \leq k<j, \\
\left|\left[B_{1}^{-1}\right]_{j, k}\right| \cdot\left|\left[B_{2}^{-1}\right]_{i-k, 1}\right| \leq c_{1} c_{2} q_{1}^{k-j} q_{2}^{i-k-1} \text { for } j \leq k<i,
\end{array}\right.
$$

where we applied the bounds for the inverse also to these terms. Since

$$
\boldsymbol{u}^{H} B^{-1} \boldsymbol{u}=\alpha^{2}\left(\left[B_{1}^{-1}\right]_{k, k}+\left[B_{2}^{-1}\right]_{1,1}\right),
$$

we further find

$$
1+\boldsymbol{u}^{H} B^{-1} \boldsymbol{u} \geq 1+\alpha^{2}\left(\frac{1}{\lambda_{\max }\left(B_{1}\right)}+\frac{1}{\lambda_{\max }\left(B_{2}\right)}\right) .
$$

Putting all these inequalities together gives the desired result.

We discuss this theorem in the following remarks.

REMARK 4.2. The result of Theorem 4.1 is based on Theorem 3.1 with $\ell=0$ for ease of presentation. It is possible to rewrite it in the spirit of (3.3) to obtain sharper decay bounds. We will state and illustrate the resulting bounds in Example 4.5 below, but refrain from giving a formal proof because it is essentially the same as that of Theorem 4.1.

REMARK 4.3. A crucial assumption in Theorem 4.1 is that $B_{1}$ and $B_{2}$ are positive definite. One situation in which this assumption is guaranteed to be fulfilled is when $A$ is strictly diagonally dominant as $B_{1}$ and $B_{2}$ inherit this property and must therefore also be positive definite.

REMARK 4.4. Theorem 4.1 only applies to the case of tridiagonal matrices, i.e., for bandwidth $\beta=1$. To modify the theorem to be applicable for matrices with bandwidth $\beta>1$, one can use a rank- $\beta$ modification that again reduces the matrix to block-diagonal form and then proceed in an analogous manner. However, the quality of the bounds obtained in this way will deteriorate more and more the larger the bandwidth is. 

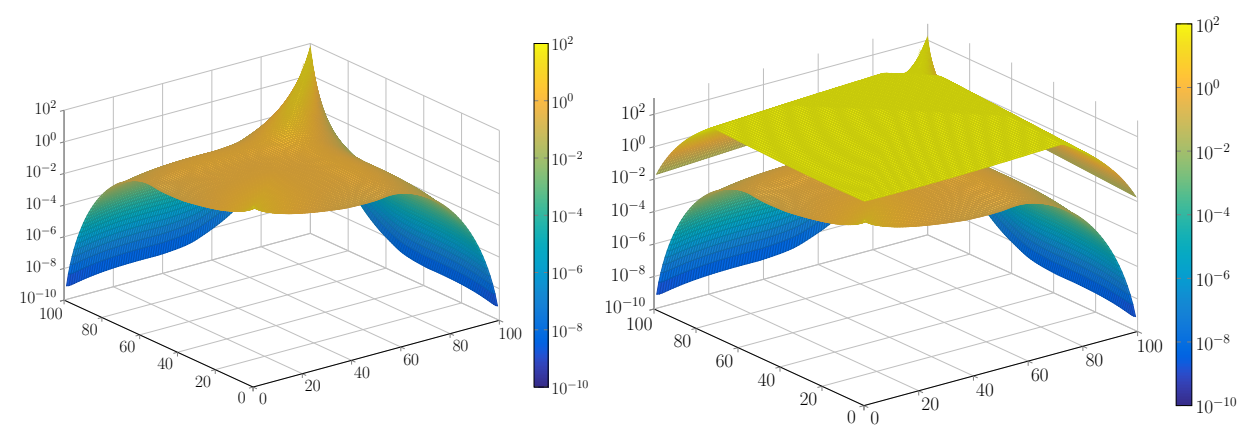

FIG. 4.1. Magnitude of the entries of $A^{-1}$ (left) and the bounds from (4.2) (right), where $A$ is the matrix from Example 1.2.

Another possible generalization of Theorem 4.1 is to consider general sparse matrices and perform the splitting by choosing a separator in the graph of $A$ and renumbering the nodes accordingly to obtain a block matrix. In this case, the exponent $|i-j|$ is replaced by $\mathrm{d}(i, j)$, the graph distance between the nodes $i$ and $j$. Again, the resulting bounds can be combined with the superlinear bounds for a general sparsity pattern according to Theorem 3.3.

Using Theorem 4.1 (modified according to Remark 4.2), we are now in a position to compute bounds that accurately predict the decay behavior of the inverse for the matrix $A$ of Example 1.2.

EXAMPLE 4.5. In this example, we illustrate the bounds arising from the blockpartitioning approach of Theorem 4.1. As Theorem 4.1 gives possibly different bounds for an entry of $\left|\left[A^{-1}\right]_{i, j}\right|$ for each value of $k$, the best possible bounds are obtained by computing bounds for every partitioning point $k=1, \ldots, n-1$ and then taking the smallest among those bounds, i.e.,

$$
\left|\left[A^{-1}\right]_{i, j}\right| \leq \min _{k=1, \ldots, n-1} Q_{i, j}^{(k)}
$$

where

$$
Q_{i, j}^{(k)}= \begin{cases}c_{1} q_{1}^{|i-j|}+c_{1}^{2} \widetilde{c} q_{1}^{2 k-j-i} & \text { for } i, j \leq k \\ c_{2} q_{2}^{|i-j|}+c_{2}^{2} \widetilde{c} q_{2}^{i+j-2(k+1)} & \text { for } i, j>k \\ c_{1} c_{2} \widetilde{c} q_{1}^{k-i} q_{2}^{j-k-1} & \text { for } i \leq k<j \\ c_{1} c_{2} \widetilde{c} q_{1}^{j-k} q_{2}^{i-k-1} & \text { for } j \leq k<i\end{cases}
$$

Note that all quantities on the right-hand side of (4.3) depend on $k$. The resulting matrix of bounds (4.2) arising for the matrix $A$ from Example 1.2 is illustrated on the right-hand side of Figure 4.1 with the magnitude of the entries of $A^{-1}$ given on the left-hand side again. We observe a considerable improvement compared to the bounds (3.3) presented in Figure 3.1. In particular, the matrix of bounds is not a Toeplitz matrix and can thus better capture the different decay rates above and below the diagonal.

Further improvements can be obtained by combining the approach of Theorem 4.1 with that of Theorem 3.1, i.e., by replacing the bounds in (4.2) by bounds involving the effective condition numbers of the diagonal blocks $B_{1}$ and $B_{2}$ for suitable values of $\ell$. The bounds obtained this way (when always selecting the best possible value $\ell$ for each entry $\left|\left[A^{-1}\right]_{i, j}\right|$ ) are depicted in Figure 4.2. These bounds resemble the actual decay behavior in $A^{-1}$ even better, and in order to allow another comparison to the results presented in Example 3.2, we show a comparison of the exact values of the first and 50th column of $A^{-1}$ with our bounds 


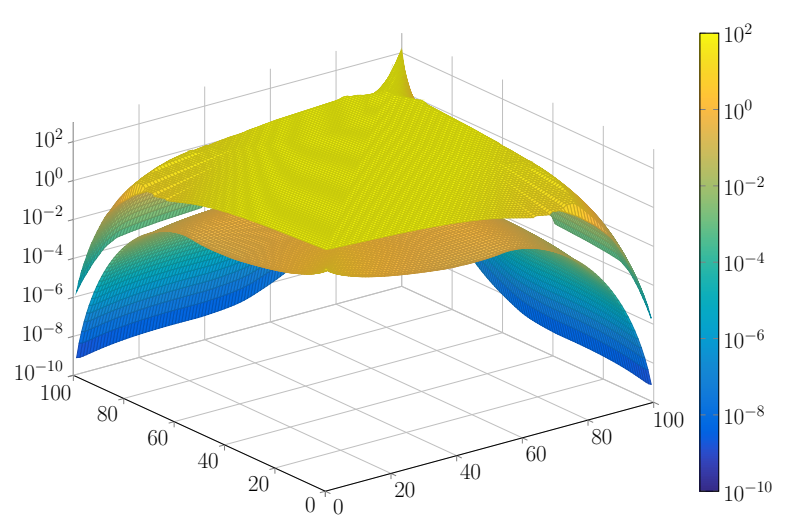

FIG. 4.2. Bounds for $A^{-1}$ obtained by combining Theorem 3.1 with Theorem 4.1, where $A$ is the matrix from Example 1.2.
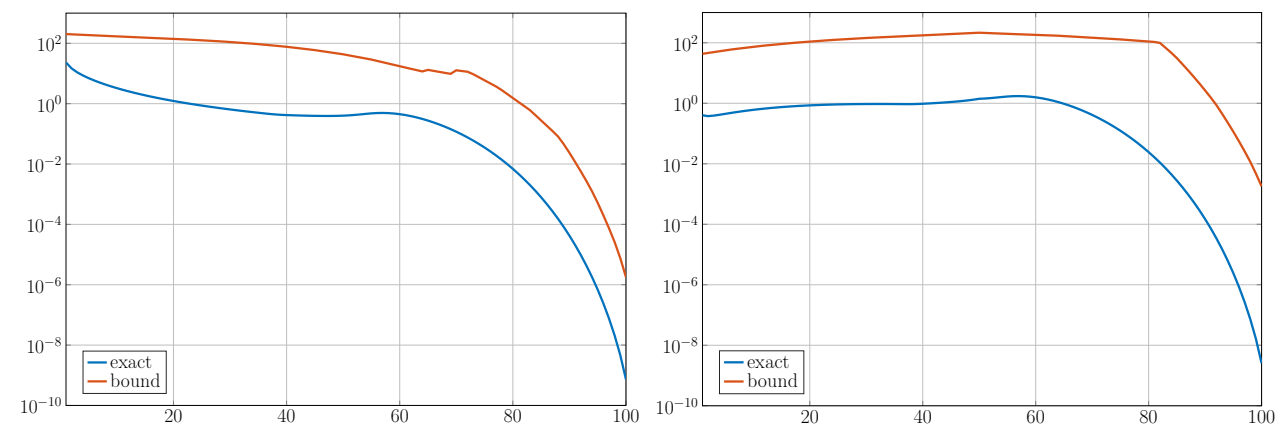

FIG. 4.3. Magnitude of the entries of the first (left) and 50th (right) column of $A^{-1}$ and corresponding bounds obtained by combining Theorem 3.1 with Theorem 4.1, where A is the matrix from Example 1.2.

in Figure 4.3. While still overestimating the entries by about two orders of magnitude, the qualitative decay behavior is resolved quite well by these bounds.

5. Conclusions. We have presented new approaches for obtaining decay bounds for the inverse $A^{-1}$ of a Hermitian positive definite, tridiagonal matrix $A$. In contrast to most bounds from the literature, these new bounds can predict superlinear decay behavior and do not obey a Toeplitz structure and can therefore more accurately predict the decay behavior in $A^{-1}$.

The main purpose of this paper was to find alternatives for obtaining decay bounds in situations where even full spectral information of $A$ does not lead to accurate results when using the classical approach from, e.g., [11]. The bounds in the presented form are not meant to be used for practical computations as they require complete spectral information of several submatrices of $A$. Finding ways to efficiently compute non-Toeplitz bounds in practical situations seems to be an interesting topic for future research.

Acknowledgment. The authors would like to thank Michele Benzi for enlightening comments on an early draft of this manuscript and Ana Šušnjara for providing the MATLAB codes used for constructing the tridiagonal test matrix.

\section{REFERENCES}

[1] R. E. BANK AND H. YSERENTANT, On the $H^{1}$-stability of the $L_{2}$-projection onto finite element spaces, Numer. Math., 126 (2014), pp. 361-381. 
[2] M. BENZI, Localization in matrix computations: theory and applications, in Exploiting Hidden Structure in Matrix Computations: Algorithms and Applications, M. Benzi and V. Simoncini, eds., vol. 2173 of Lecture Notes in Math., Springer, Cham, 2016, pp. 211-317.

[3] M. BenZI AND P. Boito, Decay properties for functions of matrices over $C^{*}$-algebras, Linear Algebra Appl., 456 (2014), pp. 174-198.

[4] M. BENZI AND G. H. GoluB, Bounds for the entries of matrix functions with applications to preconditioning, BIT, 39 (1999), pp. 417-438.

[5] M. BENZI AND N. RAZOuk, Decay bounds and $O(n)$ algorithms for approximating functions of sparse matrices, Electron. Trans. Numer. Anal., 28 (2007/08), pp. 16-39. http://etna.ricam.oeaw.ac.at/vol.28.2007-2008/pp16-39.dir/pp16-39. pdf

[6] M. BenZI AND V. Simoncini, Decay bounds for functions of Hermitian matrices with banded or Kronecker structure, SIAM J. Matrix Anal. Appl., 36 (2015), pp. 1263-1282.

[7] D. A. Bini, S. Dendievel, G. Latouche, And B. Meini, Computing the exponential of large blocktriangular block-Toeplitz matrices encountered in fluid queues, Linear Algebra Appl., 502 (2016), pp. 387-419.

[8] D. A. Bini, G. Latouche, And B. Meini, Numerical Methods for Structured Markov Chains, Oxford University Press, New York, 2005.

[9] D. R. Bowler AND T. MiYAZAKI, $O(N)$ methods in electronic structure calculations, Rep. Prog. Phys., 75 (2012), Art. 036503 (43 pages).

[10] S. DEMKO, Inverses of band matrices and local convergence of spline projections, SIAM J. Numer. Anal., 14 (1977), pp. 616-619.

[11] S. Demko, W. F. Moss, AND W. Smith, Decay rates for inverses of banded matrices, Math. Comp., 43 (1984), pp. 491-499.

[12] C. ECHEVERRÍA, J. LIESEN, AND R. NABBEN, Block diagonal dominance of matrices revisited: bounds for the norms of inverses and eigenvalue inclusion sets, Linear Algebra Appl., 553 (2018), pp. 365-383.

[13] V. Eijkhout And B. Polman, Decay rates of inverses of banded $M$-matrices that are near to Toeplitz matrices, Linear Algebra Appl., 109 (1988), pp. 247-277.

[14] R. FREUND, On polynomial approximations to $f_{a}(z)(z-a)^{-1}$ with complex $a$ and some applications to certain non-Hermitian matrices, Approx. Theory Appl., 5 (1989), pp. 15-31.

[15] A. Frommer, C. SCHIMMEL, AND M. SCHWEITZER, Bounds for the decay of the entries in inverses and Cauchy-Stieltjes functions of certain sparse, normal matrices, Numer. Linear Algebra Appl., published online 2017. https://doi.org/10.1002/nla.2131

[16] P.-L. Giscard, K. Lui, S. J. Thwaite, AND D. JaKsCh, An exact formulation of the time-ordered exponential using path-sums, J. Math. Phys., 56 (2015), Art. 053503 (18 pages).

[17] M. GU AND S. C. EISENSTAT, A divide-and-conquer algorithm for the symmetric tridiagonal eigenproblem, SIAM J. Matrix Anal. Appl., 16 (1995), pp. 172-191.

[18] A. ISERLES, How large is the exponential of a banded matrix?, New Zealand J. Math., 29 (2000), pp. 177-192.

[19] D. KERSHAW, Inequalities on the elements of the inverse of a certain tridiagonal matrix, Math. Comp., 24 (1970), pp. 155-158.

[20] D. KRESSNER AND A. ŠUŠNJARA, Fast computation of spectral projectors of banded matrices, SIAM J. Matrix Anal. Appl., 38 (2017), pp. 984-1009.

[21] J. Liesen And Z. StRakoš, Krylov Subspace Methods: Principles and Analysis, Oxford University Press, Oxford, 2013.

[22] L. LOPEZ AND A. PUgliese, Decay behaviour of functions of skew-symmetric matrices, in Proceedings of the 7th Hellenic European Conf. Computer Math. and Appl. (HERCMA 2005), E. A. Lipitakis, ed., Electronic Editions, Athens, 2005, pp. 251-256.

[23] G. Meurant, A review on the inverse of symmetric tridiagonal and block tridiagonal matrices, SIAM J. Matrix Anal. Appl., 13 (1992), pp. 707-728.

[24] R. NABBEN, Decay rates of the inverse of nonsymmetric tridiagonal and band matrices, SIAM J. Matrix Anal. Appl., 20 (1999), pp. 820-837.

[25] _ - Two-sided bounds on the inverses of diagonally dominant tridiagonal matrices, Linear Algebra Appl., 287 (1999), pp. 289-305.

[26] R. PELUSO AND T. POLITI, Some improvements for two-sided bounds on the inverse of diagonally dominant tridiagonal matrices, Linear Algebra Appl., 330 (2001), pp. 1-14.

[27] S. PozZa AND V. Simoncini, Decay bounds for non-Hermitian matrix functions, Preprint on arXiv, 2016. https://arxiv.org/abs/1605.01595

[28] Y. SAAD, Iterative Methods for Sparse Linear Systems, 2nd ed., SIAM, Philadelphia, 2003.

[29] H. R. SCHWARZ, Handbook Series Linear Algebra: Tridiagonalization of a symmetric band matrix, Numer. Math., 12 (1968), pp. 231-241.

[30] J. Sherman AND W. J. MORRISON, Adjustment of an inverse matrix corresponding to a change in one element of a given matrix, Ann. Math. Statist., 21 (1950), pp. 124-127. 